Recepción: 12/09 / 2018

Aceptación: 18 /12 / 2018

Publicación: 20 / 01/ 2019

Ciencias económicas y empresariales

Artículo de revisión

\title{
Constructivismo web: un enfoque en crecimiento para el desarrollo de la logística integral
}

\section{Constructivismo web: a focus on growth for the development of integral logistics}

\section{Constructivismo web: foco no crescimento para o desenvolvimento da logística integral}

Jessica P. Guamaquispe-Guaillaguaman ${ }^{\text {I }}$
guamanquispe.jessica7@hotmail.com

Marcelo J. Mancheno-Saá II

mj.mancheno@uta.edu.ec

Danilo J. Altamirano-Analuisa III danilojaltamirano@uta.edu.ec

Sonia F. Chaluisa-Chaluisa ${ }^{\text {VV }}$ soniach2@gmail.com

Correspondencia: guamanquispe.jessica7@hotmail.com

\footnotetext{
I Estudiante de la Universidad Técnica de Ambato, Ambato, Ecuador

II Máster Universitario en Marketing Digital y Comercio Electrónico, Magíster en Administración de Empresas Mención Planeación, Ingeniero Comercial con Mención en Marketing, Docente Universidad Técnica de Ambato, Ambato, Ecuador.

${ }^{\text {III }}$ Doctor dentro del Programa Oficial de Doctorado en Desarrollo Local y Economía Social, Máster Universitario en Economía y Desarrollo Territorial, Magíster en Gerencia Empresarial, MBA Mención Gestión De Proyectos, Ingeniero en Finanzas, Docente Universidad Técnica de Ambato, Ambato, Ecuador
}

IV Magíster en Gestión de Empresas Mención Pequeñas y Medianas Empresas, Ingeniera Comercial, Docente Universidad Técnica de Ambato, Ambato, Ecuador. 


\title{
Resumen
}

La presente investigación trata sobre la evolución de la información digital de la logística comercial e integral, el objetivo fue revisar cual es el mejor catalogo para búsquedas y las tendencias y el comportamiento de la producción científica a través de los años, a través de herramientas WEB: catálogo: Scielo, Scopus y Redalyc, además se revisó las tendencias a través de googletrends, un análisis de documentos académicos con el software publish or perish. Los resultados determinaron que el mejor catalogó para búsquedas en inglés y español de la logística integral es Redalyc ya que aporta en español bajo la palabra clave: Logística Integral con un total de 133.245 resultados y 19.834 resultados en ingles bajo el keyword: Integrated Logistic, desde el año 1999 al 2017.

Palabras claves: Gestión logística; logística integral; logística comercial; producción científica.

\begin{abstract}
This research deals with the evolution of digital information of commercial and integral logistics, the objective was to review what is the best catalog for searches and trends and the behavior of scientific production over the years, through WEB tools : catalog: Scielo, Scopus and Redalyc, in addition, the trends were reviewed through googletrends, an analysis of academic documents with publish or perish software. The results determined that the best catalog for searches in English and Spanish of the integral logistics is Redalyc since it provides in Spanish under the keyword: Integral Logistics with a total of 133,245 results and 19,834 results in English under the keyword: Integrated Logistic, from the year 1999 to 2017.
\end{abstract}

Keywords: Logistic management; integrated logistics; commercial logistics; scientific production.

\section{Resumo}

Esta pesquisa trata da evolução da informação digital de logística comercial e integral, com o objetivo de revisar qual o melhor catálogo de buscas e tendências e o comportamento da produção científica ao longo dos anos, através de ferramentas WEB.: catálogo: Scielo, Scopus e Redalyc, além disso, as tendências foram revisadas através de googletrends, uma análise de documentos acadêmicos com o software publish or perish. Os resultados determinaram que o melhor catálogo 
para pesquisas em inglês e espanhol da logística integral é Redalyc, uma vez que fornece em espanhol sob a palavra-chave: Integral Logistics com um total de 133.245 resultados e 19.834 resultados em Inglês sob a palavra-chave: Integrated Logistic, de o ano de 1999 a 2017.

Palavras-chave: Gestão logística; logística integral; logística comercial; produção científica.

\section{Introducción}

El siglo XX y el inicio del siglo XXI fue testigos de la máxima revolución digital, donde se desarrollaron de manera acelerada las telecomunicaciones y las tecnologías de información y comunicación (TIC), ahora estas nuevas tecnologías son impredecibles en el contexto social, académico y productivo, en la actualidad es difícil imaginar a las empresas o las universidades sin computadoras o internet, debido a que la sociedad del siglo XXI denominada sociedad del conocimiento en donde lo indispensable es la información, la sociedad ha evolucionado a la par con la evolución digital, la adaptación de las nuevas tecnologías a los hábitos, los procesos productivos y académicos ya no es una ventaja competitiva, ahora se ha convertido en una necesidad (Casanovas \& Cuatrecasas , 2003).

El aparecimiento de un mundo digital, de comunicaciones en línea y de inteligencia artificial, ha hecho que el esquema clásico de la interacción se rompa, tomando en cuenta que esta, forma una unidad estratégica de estudio o núcleo en las otras ciencias. Los canales de distribución de información y estrategias se han multiplicado, a lo que varios autores indican que se está viviendo una nueva etapa en la evolución del ser humano después de la revolución industrial. Los ejes principales de esta revolución fluctúan en varios ejes referenciales considerando al aprendizaje, el entretenimiento, la cultura, y la comunicación (Kroon, Swit, \& Ham, 1991).

Las generaciones que no son nativas virtuales o también conocidas como la generación X, son aquellas obligadas a superar el abismo digital a través del e-learning (Herminda, Sierra, \& Kastika, 1992). La evolución de los nuevos conocimientos es tan grande que a través de una estimación se concluye que 7.5 millones de nuevas páginas se crean diariamente, los internautas consumen el $50 \%$ del tiempo en línea para instruirse en nuevos temas, y el 90\% de personas buscan a través de motores de búsqueda, se debe recalcar que el 10\% restante es aquel que es sumamente 
especializado como para realizar búsquedas que a priori están por encima del perfil investigativo promedio (Guerra \& Aguila, 2002).

La tecnología y la industria han creado un vínculo fuerte para la generación de eficiencia, competitividad y reducción de costes operativos, Estados Unidos y España como potencias económicas referentes, se han desarrollado tanto que se han convertido en pioneros de la logística, el desarrollo explicito ha permitido un acercamiento con la tercerización, con el surgimiento de empresas especializadas en el campo de la logística, una de las ideas que evoluciona este concepto, es que los medios que permiten que el embarque, el transporte y la logística concreten como un solo término. Tomando como referencia el estudio de caso antes mencionado, se puede observar que la infraestructura antes mencionada ha permitido que la logística se lleve a cabo de manera precisa por medio de la tercerización o también conocido como outsourcing de las actividades logísticas, para disminuir costos en el corto plazo y ganar competitividad a largo plazo. (LOGINEWS, 2013).

De acuerdo a (García L. A., 2016) la internet en la actualidad crece a ritmo exponencial, cada 52 días se duplica la teoría científica contemporánea, el riesgo de tanta información está relacionada a la ambigüedad y a la inadecuada contrastación científica, haciendo que la internet sea un canal de comunicación e información rápida, pero al mismo tiempo con un gran problema porque se puede subir cualquier tipo de información sin validación científica o simplemente inventada. (LOGINEWS, 2013).

En la década de los 90, los buscadores tendían a ser analógicos con referencias de triangulación, lo que los expertos conocían como protocolos de transferencia de ficheros o FTP, pero con el tiempo y una constante evolución del término de flexibilidad se llegó a tal punto que el protocolo de transferencia HTTP (Hypertext Transfer Protocol, o protocolo de transferencia de hipertextos) se convirtió en el más utilizado en la red (Douglas, 2008).

La web se ha desarrollado tanto y de distintas maneras, que existe el internet superficial y el internet profunda o invisible, esta web también es conocida como deep web o infranet, haciendo referencia a todo aquello que los robots de los buscadores no pueden procesar haciendo que los contenidos almacenados allí sean ocultos. Generalmente este tipo de información es aquella que se oculta bajo 
protocolos explícitos o contraseñas. La cantidad de información es tan grande que existe una relación entre 500 a 1 entre lo que no se puede ver y lo que es visible en la red, ascendiendo a cerca de 600 billones de documentos ocultos en la web profunda (Rojas \& Guisao, 2011).

En relación a la logística integral, este concepto se desarrolló a partir de los años noventa, evolucionó de los conceptos básicos de la logística comercial y del marketing, ya que, al ser dos temas complementarios, pero estudiados de forma separada, la logística integral permitió romper la ideología clásica y dar a luz un nuevo término que se enfoca en una industria en desarrollo, que trata de reducir costos y tiempos para poder alcanzar la satisfacción de los clientes.

El avance de la industria permite que se desenvuelva el sistema de logística integral que no solo realiza énfasis a los conceptos básicos en cuanto a la logística comercial que fue la más reconocida y aplicada, mientras que el mix de marketing fue conocido como un sistema de aplicación que permita el ingreso exitoso de un producto en el mercado y mediante la identificación de variables: precio, plaza, producto y promoción (Ediciones Díaz de Santos, 1996).

Como referente de la logística a nivel mundial, se tiene a China, nación que se ha desarrollado de manera impresionante ya que al tener vínculos de comercio con el extranjero ha permitido que se desenvuelva en el ámbito industrial y comercial, en un principio tuvieron dificultad ya que cada empresa contaba con un departamento de logística, con el pasar del tiempo adoptaron la tercerización en la cual ingresan empresas para el abastecimiento y transporte, facilitando así la exportación de productos a todo el mundo (Oficina Económica y Comercial de la Embajada de España en Shanghai, 2010 ).

En la última década del siglo XX, según (Serra \& Kastika, 1992) la mayoría de las organizaciones no se arriesgaban a desarrollar una sistema de logística integral ya que preferían desarrollar toda la logística en sus miso departamentos lo cual en ocasiones, hacía que la presión de realizar todas las actividades, represente costos innecesarios, ya que no tenían personal capacitado, ni la experiencia, ni un control adecuado en las actividades logísticas (Ediciones Díaz de Santos, 1996)

Según (Guerra \& Águila, 2002) las organizaciones al igual que la época contemporánea hacen referencia a los insumos, materiales y recursos pero hacen relevancia a la información, recursos 
humanos, transporte, abastecimiento, almacenaje y distribución tomando en cuenta en satisfacer las necesidades de los clientes.

La logística integral y su enfoque de naturaleza clásica y evolución contemporánea, intenta optimizar las actividades operativas, tomando en cuenta la gestión de suministros, apalancándose en una actividad estratégica denominando planificación, innovando constantemente ,controlando de forma eficiente y efectiva todos los flujos directos e indirectos, relacionando los puntos de almacenaje y distribución, para una adecuada satisfacción de necesidades pero tomando en cuenta la contratación de servicios terceros que aportan para su adecuada distribución en el tiempo adecuado y con menos costos (Anaya, 2016).

Con el tiempo y varias revisiones literarias, congresos y reuniones de expertos, diversificación de temas, concepción de variables y subvariables se ha hecho referencia a términos de naturaleza modernista como el outsourcing o externalización que representa un anclaje estratégico para el adecuado desarrollo de las actividades tanto internas como externas. (Montoya, Torres, Vidal, \& Marrnolejo, 2013) mencionan que las empresas subcontratan almacenaje, bodegas, enfriadoras, transporte y distribución, esto permite a las organizaciones optimicen tiempo ya que la distribución es más efectiva en el menor tiempo.

En el desarrollo de la logística en América Latina se nota índices de gestión inadecuada que se traduce en la demora en los procesos logísticos ya que al realizar importaciones y exportaciones no se aplica adecuadamente la logística, los departamentos de logística no cuenta con personal capacitado que sepan sobre procedimientos, así también el grado de industrialización son factores que afectan las operaciones logísticas empresariales. (Banco de Desarrollo de América Latina, 2014).

La logística integral se estudia desde la subcontratación u outsourcing ya que la literatura demuestra que contratar a una empresa especializada en logística integral permitirá desarrollar un plus en las empresas ya que la contratar a una empresa con experiencia en el sector permitirá tener ventajas competitivas en costos y permitir los productos de una empresa adecuadamente en el punto de entrega en cualquier mercado a nivel mundial (Rojas, Guisao, \& Cano, 2011). Métodos 
Se utilizó un estudio descriptivo transversal mediante el análisis bibliométrico de todos los artículos científicos, páginas web, tesis de pregrado y posgrado que se encuentren disponibles en la web, tanto en idioma español e inglés, mediante palabras claves en español: logística integral, gestión logística y logística comercial y en ingles con las palabras clave: integral logistics, logistics management and commercial logistics.

Para obtener la base de datos se utilizó la base de datos de Scielo, Scopus y Redalyc y mediante SEO (Search Engine Optimization o también conocido como Optimización para motores de búsqueda). La investigación se realizó durante el periodo comprendido entre: octubre 2018 y la última búsqueda se efectuó el 10 de diciembre del 2018.

Se destacan tres descriptivos dentro del análisis bibliométrico teoría logística integral: 1. el idioma, 2. las keywords relacionadas a la teoría y sus variables, las cuales pudieron también ser comparadas de acuerdo al patrón de búsqueda en los diferentes buscadores y 3. La evolución por zonas, indicando en que sectores geográficos.

El criterio empleado para la selección de documentos estaba direccionado a los aportes más relevantes a través del tiempo, así mismo se organizó dichos documentos mediante el software Publish or Perish Versión: 6.45.6354 que permitió identificar por el volumen de teoría cronológica el inicio oficial del término en estudio a través de un cruce en tendencia, se determinó que en habla hispana nace en 1990 y en habla inglesa en 1995.

El procedimiento se basó en la búsqueda y descarga de los artículos disponibles en los sitios de indexación Redalyc, Scopus y Scielo, además se realizó una base de datos en la cual se registró la información correspondiente a los resultados y características de cada base de indexación.

\section{Resultados}

Se muestra los resultados de la búsqueda realizada mediante el software Publish or Perish sobre los libros y artículos más relevante sobre la logística integral.

Tabla 1 Revisión bibliográfica más relevante sobre la logística integral 


\section{Logística Integral}

\begin{tabular}{|c|c|c|c|c|c|}
\hline \multicolumn{6}{|c|}{ Cronología Referencia Bibliográfica } \\
\hline $\begin{array}{c}\mathbf{N} \\
0\end{array}$ & LIBRO / Revista & Año & Autor & Aporte a la Teoría & Keywords \\
\hline 1 & $\begin{array}{l}\text { Administración y } \\
\text { estrategia }\end{array}$ & 1992 & $\begin{array}{l}\text { Herminda, } \\
\text { Serra \& } \\
\text { Kastika }\end{array}$ & $\begin{array}{l}\text { Logística Integral realiza una } \\
\text { referencia a los insumos, recursos } \\
\text { que se necesita para la distribución, } \\
\text { realizando énfasis en el entorno. }\end{array}$ & $\begin{array}{l}\text { Insumos, } \\
\text { Recursos }\end{array}$ \\
\hline 2 & $\begin{array}{l}\text { Compras } \\
\text { Inventarios }\end{array}$ & 1996 & $\begin{array}{l}\text { Ediciones } \\
\text { Días de } \\
\text { Santos S.A, }\end{array}$ & $\begin{array}{l}\text { Permite satisfacer las necesidades de } \\
\text { los clientes en el menor tiempo } \\
\text { posible, en el cual se realiza una } \\
\text { planificación y programación con } \\
\text { enfoque al entorno externo e interno. }\end{array}$ & Programación \\
\hline 3 & $\begin{array}{l}\text { La planificación } \\
\text { Estratégica en el } \\
\text { Agro negoció }\end{array}$ & 2002 & & $\begin{array}{l}\text { Se desarrolla una estrategia para los } \\
\text { productos sustitutos, para un } \\
\text { adecuado almacenamiento de los } \\
\text { productos, basado en un adecuado } \\
\text { proceso de abastecimiento. }\end{array}$ & Insumos, Proceso \\
\hline 4 & Logística Integral & 2003 & $\begin{array}{l}\text { Casanovas } \\
\text { A. \& \& } \\
\text { Cuatrecasas }\end{array}$ & $\begin{array}{l}\text { Se define por la reacción de los } \\
\text { clientes , importante para las } \\
\text { empresas en la época industrial } \\
\text { donde se adopta este tipo de sistema. }\end{array}$ & Sistema \\
\hline 5 & $\begin{array}{l}\text { Ingeniería } \\
\text { "Logística } \\
\text { Reserva Retos } \\
\text { para la Ingeniería } \\
\text { Industrial " }\end{array}$ & 2005 & $\begin{array}{l}\text { Néstor } \\
\text { Monroy \& } \\
\text { Ahumada } \\
\text { María } \\
\text { Claudia }\end{array}$ & $\begin{array}{l}\text { Desarrolla y optimiza actividades } \\
\text { operativas en el cual realiza énfasis } \\
\text { en la manufactura, distribución, } \\
\text { consumo, post- consumo }\end{array}$ & Distribución \\
\hline 6 & $\begin{array}{lr}\text { "Manual básico } \\
\text { de } & \text { logística } \\
\text { integral" } & \end{array}$ & 2006 & $\begin{array}{l}\text { Aitor Urzelai } \\
\text { Inza }\end{array}$ & $\begin{array}{l}\text { Se define Logística integral como } \\
\text { parte de la gestión de la cadena de } \\
\text { suministros encargada de planificar, } \\
\text { implementar y controlar de forma } \\
\text { eficiente y efectiva el almacenaje y } \\
\text { flujo directo e inversa de los bienes, } \\
\text { servicios }\end{array}$ & $\begin{array}{l}\text { Planificación, } \\
\text { implementación y } \\
\text { control }\end{array}$ \\
\hline 7 & $\begin{array}{l}\text { Universidad } \\
\text { EAN" Gerencia } \\
\text { Lógica y Global " }\end{array}$ & 2009 & $\begin{array}{l}\text { Pablo Cesar } \\
\text { Ocampo } \\
\text { Vélez }\end{array}$ & $\begin{array}{l}\text { Proceso eficaz y eficiente que busca } \\
\text { un equilibrio entre el nivel de } \\
\text { servicio y costos reduciendo los } \\
\text { mismos. }\end{array}$ & $\begin{array}{l}\text { Eficacia } \\
\text { Eficiencia }\end{array}$ \\
\hline 8 & $\begin{array}{ll}\text { Logística } & \text { Integral } \\
\text { La } & \text { gestión } \\
\text { operativa } & \text { de la } \\
\text { empresa } & \end{array}$ & 2011 & $\begin{array}{l}\text { Julio Juan } \\
\text { Anaya } \\
\text { Telero }\end{array}$ & $\begin{array}{l}\text { Constituye una visión de conjunto, } \\
\text { no profundiza demasiado los } \\
\text { problemas técnicos específicos, } \\
\text { basada en una filosofia de control } \\
\text { del flujo de materiales. }\end{array}$ & Control de Flujos \\
\hline 9 & $\begin{array}{l}\text { Logística integral } \\
\text { "Una propuesta } \\
\text { practica para su }\end{array}$ & 2011 & $\begin{array}{l}\text { Rojas, } \\
\text { Guisao }\end{array}$ & $\begin{array}{l}\text { Se define como el control del flujo } \\
\text { de materiales así como información } \\
\text { de fuentes de aprovisionamiento }\end{array}$ & $\begin{array}{l}\text { Control de flujos } \\
\text { y } \\
\text { aprovisionamiento }\end{array}$ \\
\hline
\end{tabular}


10 Logística Gestión de la cadena de suministros

11 Nova Scientia " Logística Integral; Una alternativa para crear valor y ventaja competitiva en las pequeñas mediana empresas (pymes) Sector del Calzado.

12 Entramado " La logística de reserva y su relación con la gestión integral y sostenible de residuos sólidos de sectores productivos."

13 Logística Integral "La gestión Operativa de la empresa" Council of Logistics Management 1986

14 Semestre Económico: "Contratación Logística en Colombia: Implementación de un operador Logístico Integral"

15 Universidad Tecnológico de Pereira " Modelo de capacitación sobre lógica integral de almacenamiento para autoservicios de retail"
2012 Lluis

Cuatrecasas

Arbos

\section{Villareal}

Segoviano

Francisco

Javier

\section{3}

Montoya, Torres, Vidal

\&

Marrnolejo

2015

\section{Julio}

Anaya

Telero

2015

Gonzalo Correa, John Alexander

\section{Ballesteros \\ Pedro, \\ Robledo \\ Mauricio \& \\ Barrios \\ Héctor}

Comprende una integración de planificación, organizar y controlar las actividades relacionadas con traslado y almacenamiento de materiales

Se aplica este sistema de logística integral para incrementar estrategias y así permite un creciente valor agregado a la organización, así como una adecuada calidad de producto, distribución y apoyo al cliente.
Planificar, organizar, controlar

Estrategias, valor agregado
Transferencias,

Basada en el Proceso de asociar la generación, manipulación, almacenamiento, recolección, transferencia, trasporte, procesamiento, y la habilidad de involucrar trabajos interdisciplinarios.

$<$ proceso de planificación, implementación y control eficiente del flujo efectivo de costes y almacenaje de materiales, inventarios en curso y productos terminados, así como la información relacionada desde el punto de origen punto de consumo con el fin de atender necesidades del cliente $>>$

El sistema de logística integral es de gran ayuda para la empresa ya que resume diferentes actividades de almacenaje para la producción.

Logística integral incorpora algunos elementos para la manipulación, distribución, almacenamiento de productos hasta el momento en que es entregado al cliente planificación, control, flujo de efectivo, materiales

Sistema, abastecimiento, optimización. 


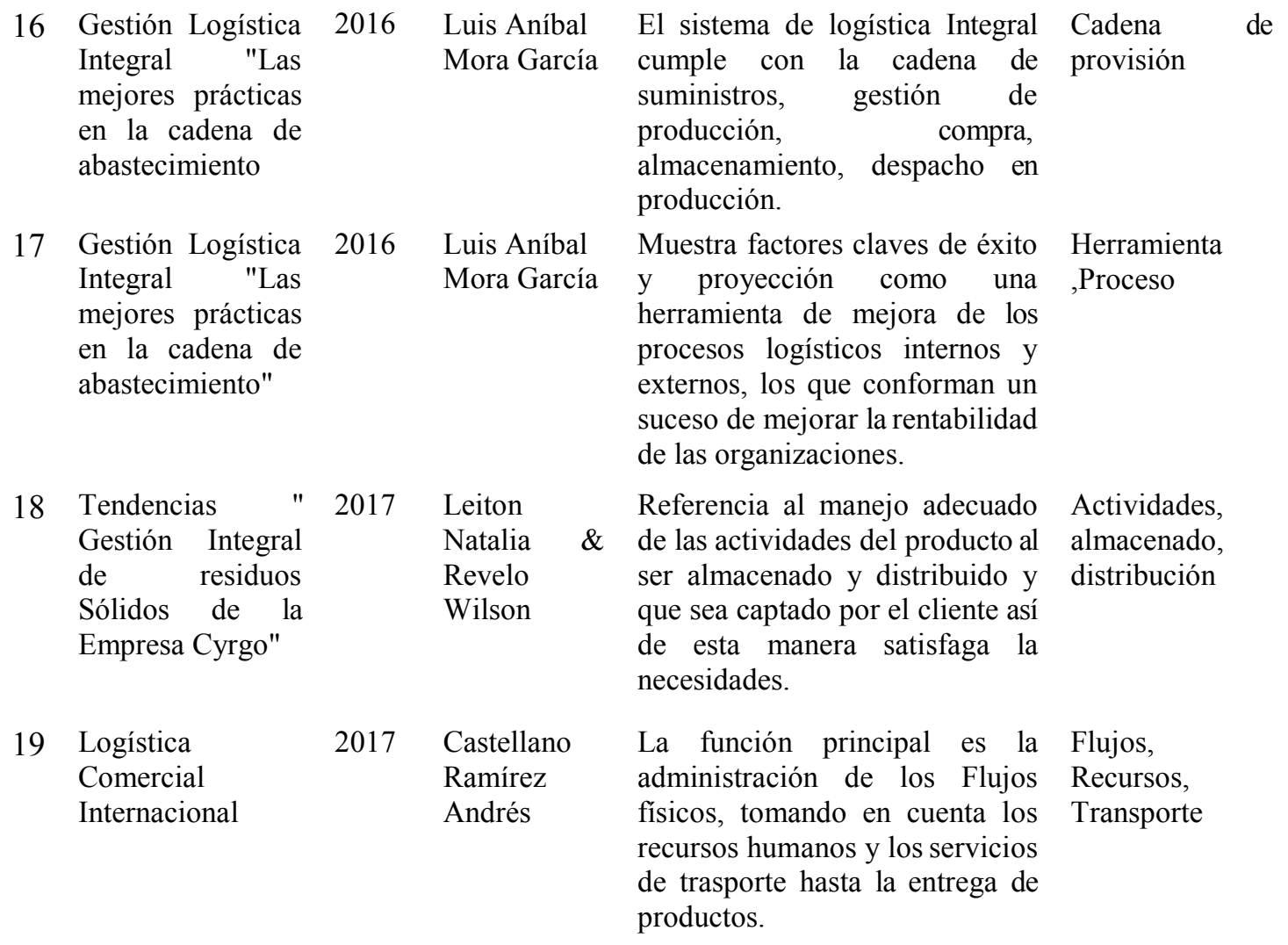

\section{Fuente: Revisión bibliográfica \\ Elaborado por: Autores}

En la tabla 1, se puede observar como las palabras clave o keywords han cambiado con el pasar de los años, han evolucionado de forma que se observa como se ha modificado ciertos términos y se han asociado a la logística integral, estos han sido gran ayuda para desarrollar conceptos de un término de logística integral, al relacionar estas palabras tienen relación entre ellas para dar una mejor definición así en el año 1992 las palabras Insumos y recursos que en los años noventa eran más reconocidos para interpretar los en los procesos de producción seguidos en el año de 1996 con la programación de tareas en el 2002 Materiales y los procesos y en el 2003 la palabra de sistema en el 2005 Distribución al año 2006 lo relacionamos con planificación implementación y control, en el 2009 la eficiencia y la eficacia se suman a este término al 2011 control de flujos y el Aprovisionamiento, en el año 2012 adjuntamos las estrategia y el valor agregado, en el año 2013 transferencia y habilidades, en el año 2015 encontramos el termino de flujo de efectivo y optimización, 2016 la Cadena de Provisiones y herramientas la más conocidas en un proceso de producción, en el año 2017 lo relacionamos con las Actividades de almacenamiento y distribución y transporte. 


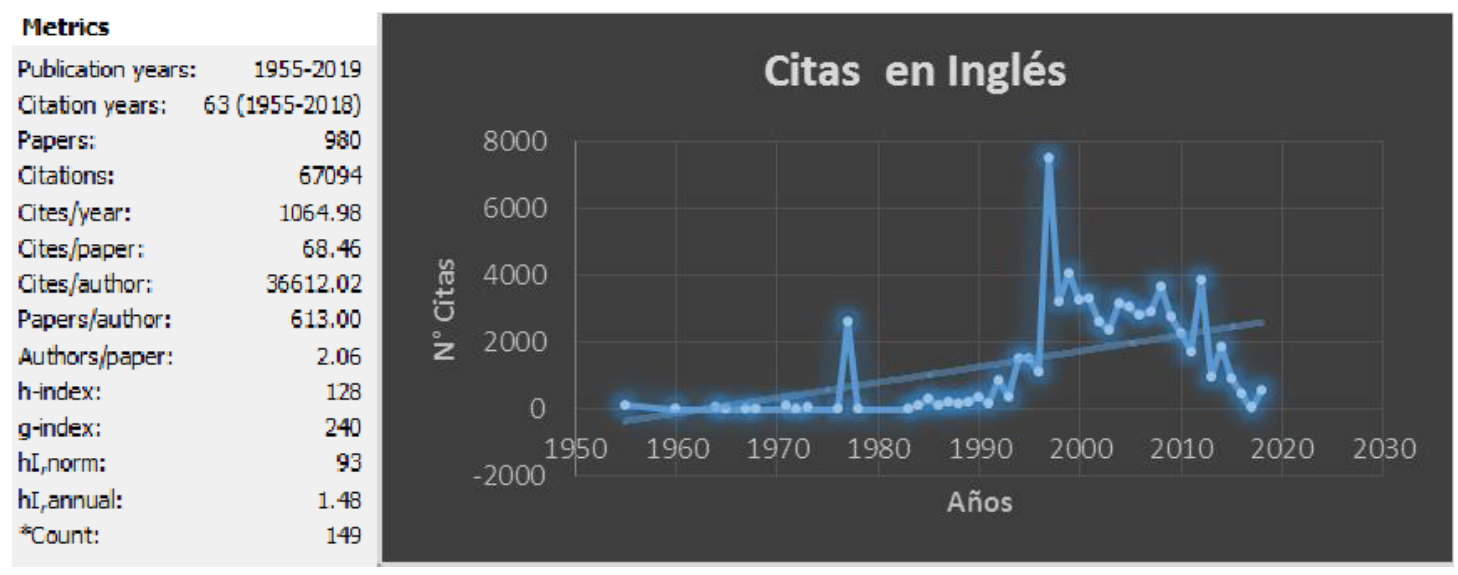

Gráfico 1 Citas en inglés

Elaborado por: Autores

Al hacer un análisis descriptivo de la información obtenida en el software Publish or Perish se puede ver que la teoría del término de logística integral ha sido estudiado a manera de secuencia desde mediados del siglo XX, en la cual se observan que la información de citas en ingles empiezan en el año de 1955 en el cual, haciendo referencia a la tendencia y evolución existente se debía haber empezado 10 años antes, se entiende que existió en su inicio una década en la cual la logística pasó de ser un término meramente militar a tener concepciones comerciales y empresariales. A finales del año 2000 referenciado al siglo XX, se ve un pico importante antes de cerrar el siglo por lo que podría considerarse que la logística modernista recién inicia en el siglo XXI. Si se considera en el estudio 64 años de investigación, alrededor de dos autores promedio por artículo, se hace referencia que el tema, es ampliamente estudiado y que con el tiempo la concepción del mismo ha evolucionado tanto que hace referencia a un concepto integral que va mucho más allá de la logística militar.

Guerra G. \& Águila A. 


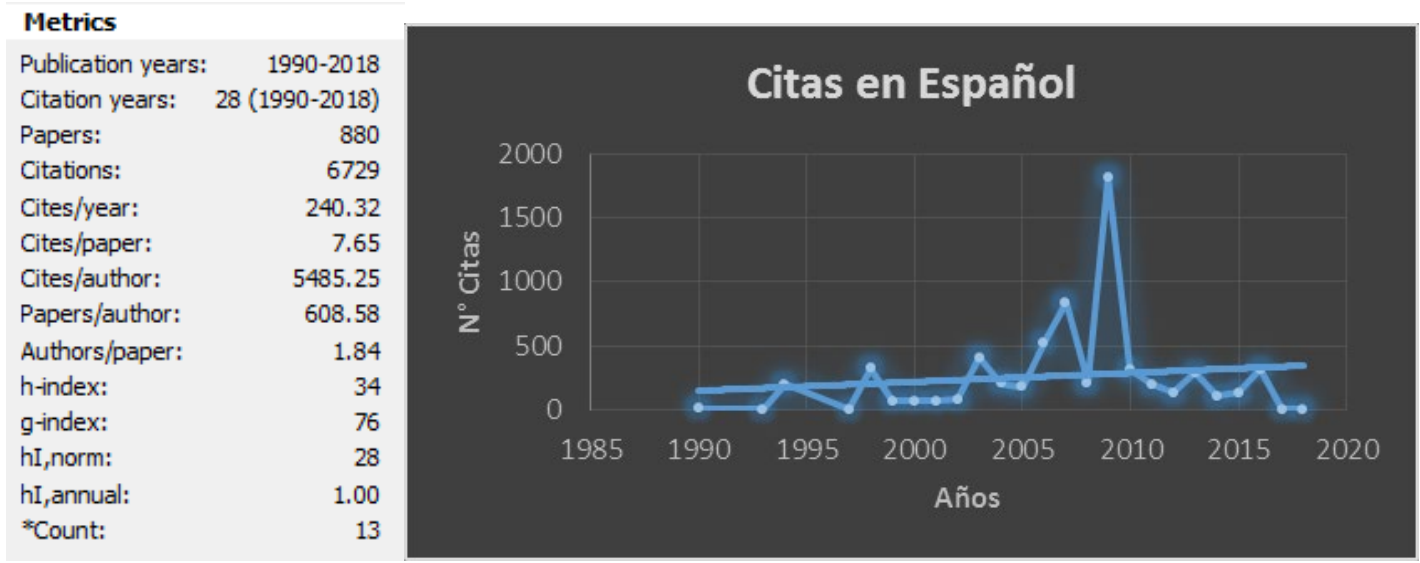

Gráfico 2 Citas en español

Elaborado por: Autores

Al haber realizado un estudio de las publicaciones de habla hispana sobre logística integral se puede observar que la ciencia en el idioma de estudio aparece en la década de los años 90 donde le toma alrededor de 4 años para expandirse y formalizarse en el campo, la utilización de este estudio referencia 7 citas por paper indicando que la teoría se vuelve dispersa y la representatividad del material científico no es muy alto. Uno de los picos más altos se referencia en el año 2009, sin embargo, este dato descriptivo representa únicamente la cuarta parte de los estudios en habla inglesa.

El SEO es una herramienta que permite que las keywords estén encaminadas a la optimización adecuada, siendo esta encontrada o etiqueta por los buscadores más reconocidos no solo permite que se posesione en la web, al mismo instante registra el número de frecuencia con la cual ingresa esta etiqueta y da los resultados de la búsqueda favorable a la persona que lo busca, en el cual los motores de búsqueda juegan un papel importante dentro de la búsqueda en el cual recomiendan que el contenido sea preciso y de fácil acceso de carga, esto permite obtener rápidamente la información. (Ramos, 2012)

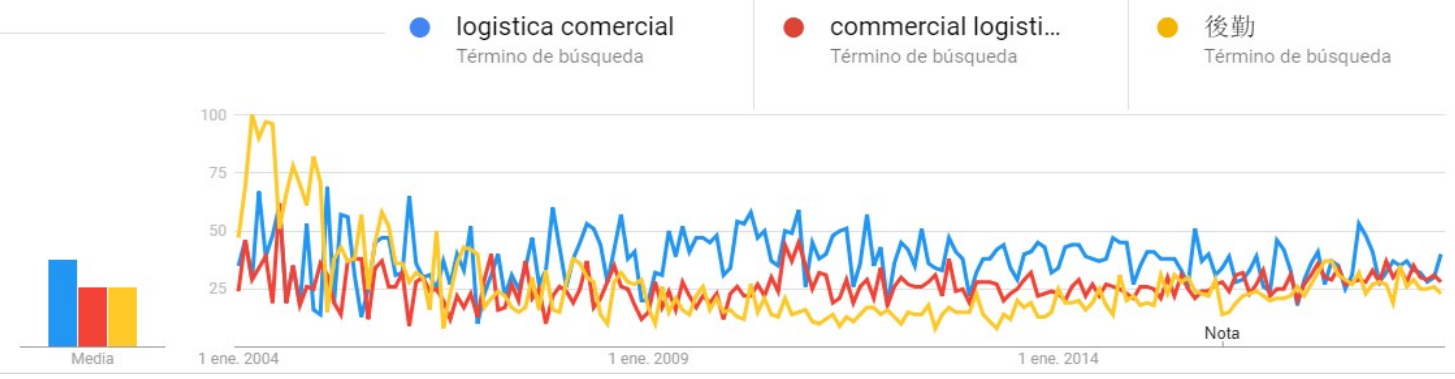



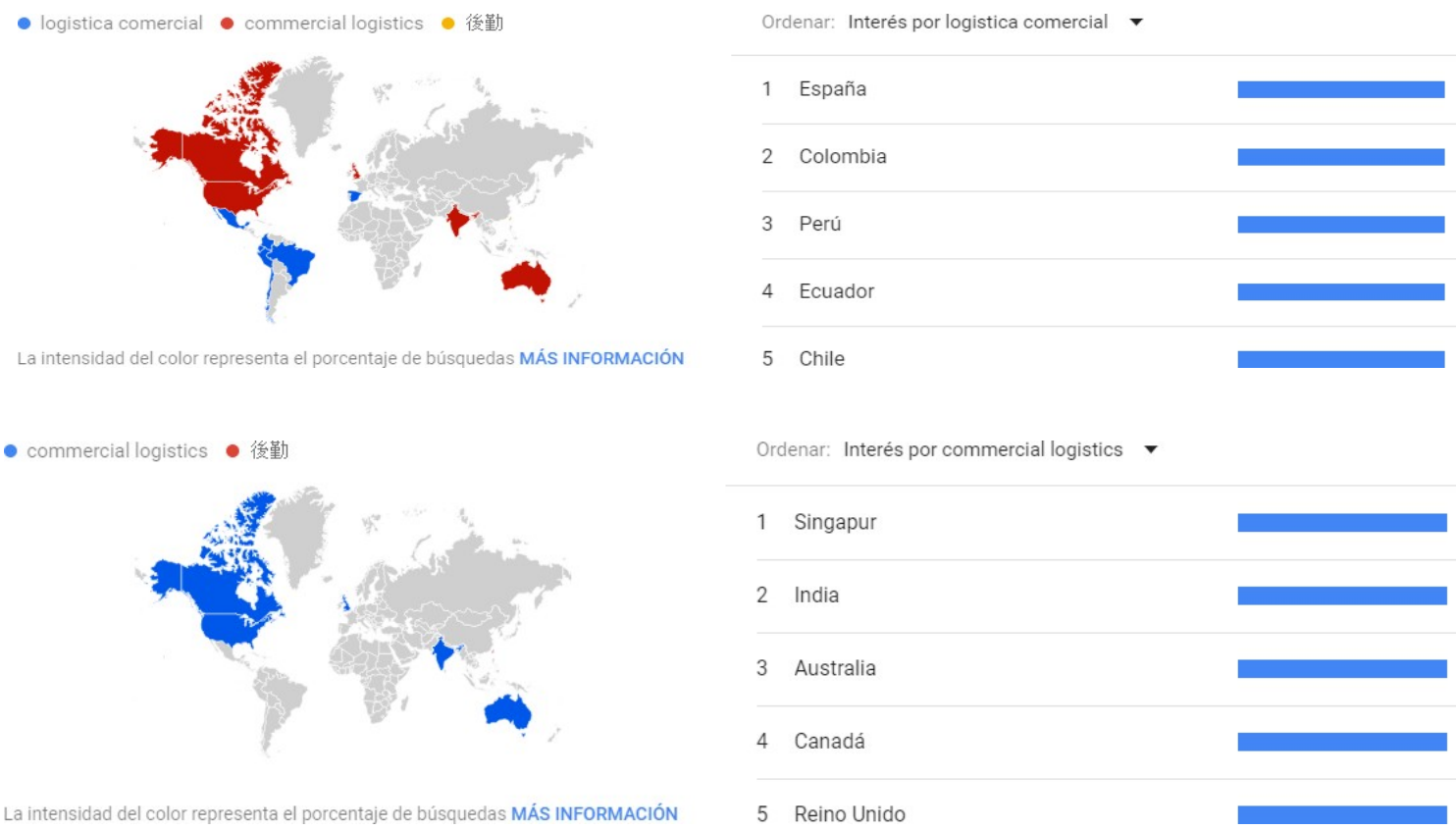

La intensidad del color representa el porcentaje de búsquedas MÁs INFORMACIÓN

Gráfico 3 SEO de los términos logística comercial Elaborado por: Autores

Mediante la herramienta SEO se determinó que la logística comercial en español tiene mayor interés en: España, Colombia, Perú, Ecuador y Chile. Mientras que comercial logistics en ingles tiene más interés en Singapur, India, Australia, Canadá y Reino Unido. Se puede indicar que tanto España y Singapur lideran el interés por la logística a nivel mundial.

Tabla 2 Revisión base de datos: Scielo, Scopus y Redalyc

\begin{tabular}{ccc}
\hline Artículos & Total & Porcentaje \\
\hline & Palabra clave & \\
& Logística integral & \\
& 103 Resultados & \\
& Idiomas: & \\
& Español: 57 & \\
& Inglés: 32 & \\
& Portugués: 31 & $0.06 \%$ \\
& Tipo de publicación & \\
Scielo: & Artículos: 99 & \\
Método: Integral & Editorial: 2 & \\
Nivel regional & Otros: 2 & \\
& Años: & \\
& $1997-2018$ & \\
& Países: & \\
& Brasil: 27 & $0.03 \%$ \\
& Colombia: 17 & \\
& México: 14 & \\
& Cuba: 8 & \\
& España: 6 & \\
\hline
\end{tabular}


Constructivismo web: un enfoque en crecimiento para el desarrollo de la logística integral

\begin{tabular}{|c|c|c|}
\hline $\begin{array}{l}\text { Método: Integral } \\
\text { Nivel regional }\end{array}$ & $\begin{array}{l}\text { Integrated logistics } \\
47 \text { resultados } \\
\text { Idiomas: } \\
\text { Inglés: } 19 \\
\text { Portugués: } 17 \\
\text { Español: } 12 \\
\text { Tipo de publicación } \\
\text { Artículos: } 45 \\
\text { Artículo de revisión: } 2 \\
\text { Países: } \\
\text { Brasil: } 37 \\
\text { México: } 8 \\
\text { Colombia: } 7 \\
\text { España: } 5 \\
\text { Sudáfrica: } 4\end{array}$ & \\
\hline $\begin{array}{c}\text { Scopus } \\
\text { Método: Display only Open } \\
\text { Access journals, }\end{array}$ & $\begin{array}{l}\text { Keyword } \\
\text { Integrated logistics } \\
21 \text { resultados } \\
\text { Idiomas: } \\
\text { Inglés: } 21 \\
\text { Tipo de publicación } \\
\text { Revistas: } 19 \\
\text { Congresos: } 1 \\
\text { Publicaciones comerciales: } 1\end{array}$ & $0.01 \%$ \\
\hline Redalyc & $\begin{array}{l}\text { Palabra clave } \\
\text { Logística integral } \\
132245 \text { Resultados } \\
\text { Años: } 1999-2015 \\
\text { Idiomas: } \\
\text { Español: } 89565 \\
\text { Portugués: } 33022 \\
\text { Inglés: } 9195 \\
\text { Países } \\
\text { Brasil: } 36214 \\
\text { Colombia: } 21900 \\
\text { México: } 21784 \\
\text { España: } 13536 \\
\text { Cuba: } 7783 \\
\text { Ecuador: } 1232\end{array}$ & $75.49 \%$ \\
\hline Redalyc & $\begin{array}{l}\text { Keyword } \\
\text { Integrated logistics } \\
42759 \text { Resultados } \\
\text { Años: } 1999-2017 \\
\text { Idiomas: } \\
\text { Inglés: } 19834 \\
\text { Español: } 15671 \\
\text { Portugués: } 7025 \\
\text { Países } \\
\text { Brasil: } 14132 \\
\text { México: } 7319 \\
\text { España: } 6182 \\
\text { Colombia: } 6061 \\
\text { Ecuador: } 1232\end{array}$ & $24.41 \%$ \\
\hline TOTAL & 175.175 artículos & $100.00 \%$ \\
\hline
\end{tabular}


Elaborado por: Autores

Evolución por años en base Scielo: Logística Integral

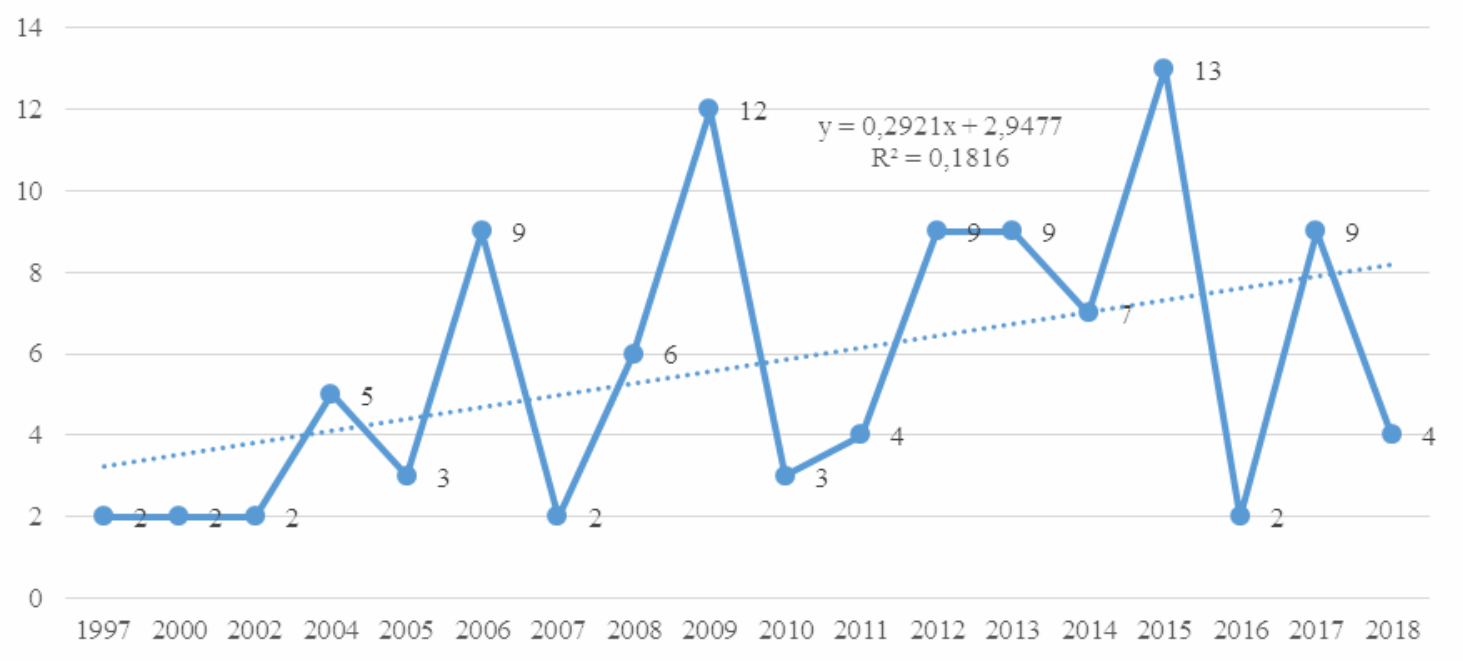

Gráfico 4 Evolución por años Scielo: Logística Integral Elaborado por: Autores

El término en español logística integral, en base de datos de Scielo se originó por primera vez en 1997, el año de mayor publicación es 2015 con 13 artículos, se puede observar que hay un decrecimiento en los números de publicaciones.

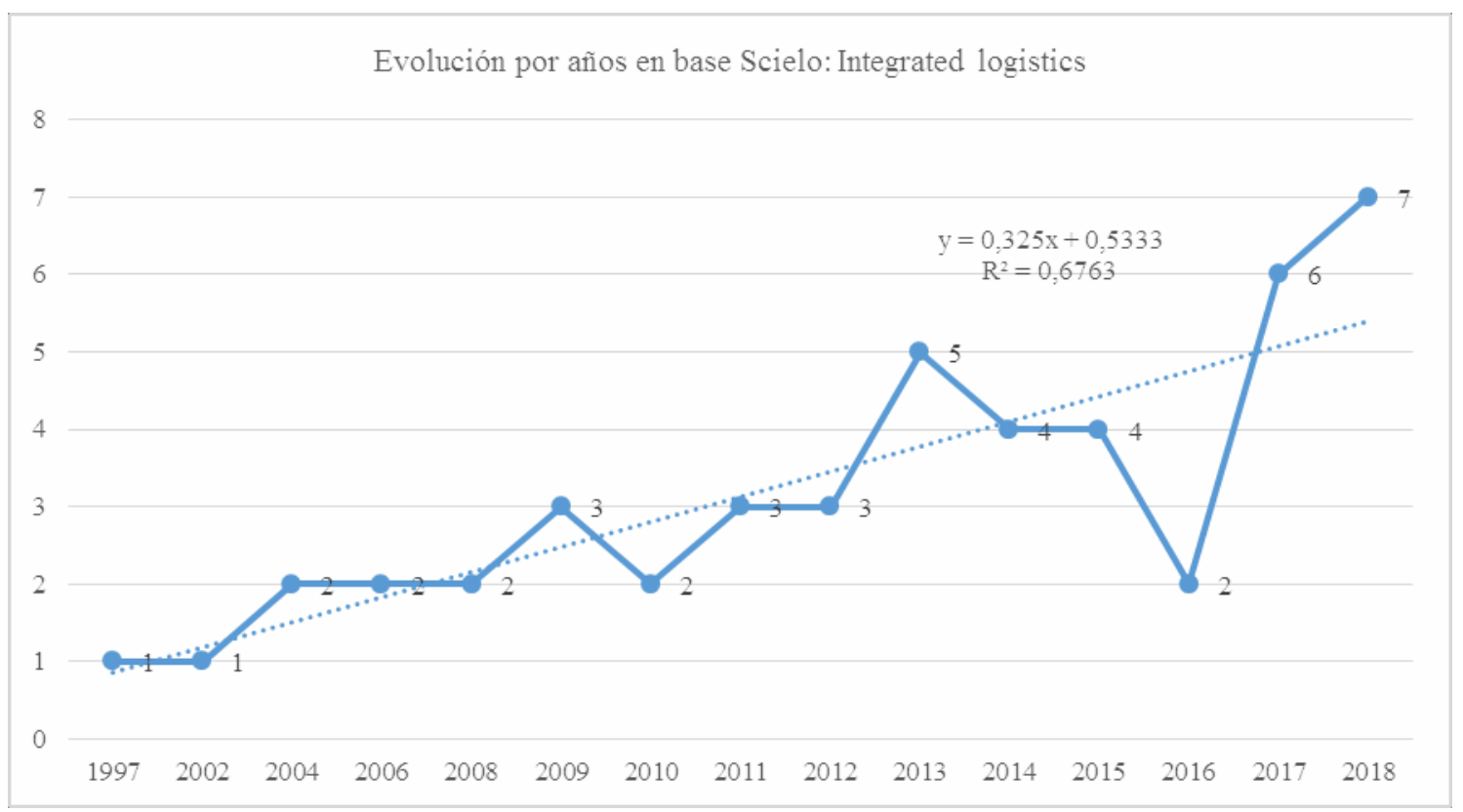

Gráfico 5 Evolución por años Scielo: Integrated logistics Elaborado por: Autores 
El termino en inglés: integrated logistics, en base de datos de Scielo se originó por primera vez en 1997, el año de mayor publicación es 2018 con 7 artículos, se observa una tendencia positiva en el aumento de publicaciones.

\section{DISCUSIONES}

- El termino logística integral tuvo su origen en los años 90, se deriva de las teorías clásicas de la logística y el marketing, ha evolucionado a la par con el concepto de tercerización o también conocido como el outsourcing debido a la aparición de empresas especialistas en logística integral que permiten a través de sus servicios, experiencia y eficiencia que reduce costes, mejora la competitividad y permite colocar los productos en cualquier mercado internacional.

- El catalogo indexado que mayor aporta a la evolución teórica y científica es Redalyc, ya que aporta el 75.49\% de las búsquedas en español: bajo los términos Logística integral y el $24.41 \%$ bajo los términos en inglés: Integrated logistics.

- En relación a los años de publicaciones, en Scielo: bajo la búsqueda: Logística comercial, existen registros desde el año 1997 hasta el año 2018, con un total de 103 artículos, el año de mayor producción científica fue el año 2015 con 13 artículos, en relación a la búsqueda:, la tenencia es negativa para la producción de artículos científicos en español Integrated logistics se tiene registros desde el año 1997 hasta el 2018, con una tendencia positiva a la producción científica en idioma inglés.

\section{Recomendaciones}

- Una de las restricciones fue el acceso a la InCites Journal Citation Reports JCR de Thompson Reuter, debido a que se necesitaba de un usuario y una contraseña para poder ingresar a los índices de impactos y sus variables, por lo tanto, se recomienda que la Universidad Técnica de Ambato pueda realizar la gestión para habilitar el acceso a sus estudiantes.

\section{Referencias Bibliográficas}

Anaya Tejero, J. (2011). Logistica Integral " La gestion operativa de la empresa" (4ta ed.). Madrid.

Anaya, J. (2016). Logistica Integral " La gestion operativa de la empresa " (5a ed.). Bogotá: Editorial ESIC. 
Banco de Desarrollo de Améria Latina . (2014). Programa de Desarrollo Logístico CAF LOGRA. Desarrollo Logístico Regional en Latinoamérica, 8- 40 .

Carrasco, J. (2000). Evolución de los enfoques y conceptos de la Logística Su impacto en la dirección y gestión de las organizaciones. Economia Indutrial, 5 - 18.

Casanovas , A., \& Cuatrecasas . (2003). Logica Integral .

Castellano Ramírez, A. (2017). Logística Comercial Internacional . Colombia.

Cuatrecasas, \& Casanovas. (2003). Logistica Integral. Barcelona.

Cuatrecasas, L. (2012). Logistica Gestion de la cadena de suministros. Madrid.

Douglas, L. M. (2008). Supply Chain Management: Processes, Partnership, Performance (3ra ed.). United States of America.

Ediciones Diaz de Santos. (1996). Compras e Inventarios. Madrid: EditorialDiaz de Santos.

Garcia, L. (2016). Gestión Logística Integral" Las prácticas en la cadena de abastecieminto" (3ra ed.). Bogota.

Garcia, L. A. (2016). Gestión Lógica Ingretal " Las mejores practicas en la cadena de abastecimiento (2da ed.). Bogota.

Gonzalo, C. J. (2015). Semestre Economico. Contratación Logística en Colombia: Implementacion de un operdor Logistica Integral, 225.

Guerra, G., \& Aguila, A. (2002). La planificacion Estrategica en el Agro negocio. Mexico.

Herminda, Sierra, \& Kastika. (1992). Administración y Estrategia. Argentina.

Inza, A. U. (2009). Manual Basico de logistica Integral. Colombia.

Kroon, M., Swit, R., \& Ham, J. v. (1991). Freight transport and the enviroment . London . 
Leiton, N., \& Revelo, W. (2017). Gestión Integral de residuos Sólidos de la Empresa Cygo. Tendencias.

LOGINEWS. (24 de Abril de 2013). Chicago la zona mas atractiva en USA para el sector Logistico . LOGINEWS.

Montoya, Torres, Vidal, \& Marrnolejo. (2013). La logistica de reserva y su relacion con la gestion integral y sostenible de residuos solidos de sectores productivos. Entramado, 226-238.

Mora García, L. A. (2016). Las mejore prácticas en la cadena de abastecimiento. Gestión Logística Integral .

Nestor, M., \& Ahumada, M. (2005). Logistica Reserva retos para la Ingenieria Industrial. Ingenieria, 25.

Oficina Económica y Comercial de la Embajada de España en Shanghai. (2010 ). El sector de la Logistica en China . Estudios de Mercado .

Pedro, B., Mauro, R., \& Hector, B. (2015). Modelo de capacitacion sobre logistica integral de almacenamiento para autoservicios de retail. Universidad Tecnologico de Pereira, 32.

Ramírez, C. C. (2004). La gestión administrativa en las instituciones educativas. México.

Ramos, J. (2012). SEO; Guía práctica de posicionamiento en buscadores . Autor-Editor .

Rojas, \& Guisao. (2011). Logistica Integral Una propuesta practica para su negocio. Bogotá.

Rojas, M., Guisao, E., \& Cano, J. (2011). Una propuesta práctica para su negocio. Bogotá: Ediciones de la $\mathrm{U}$.

Serra, H., \& Kastika. (1992). Administracion y estratégica.

Telero, A. J. (2015). Logistica integral " La gestion Operativa de la Empresa (5ta ed.). Madrid.

Velez, O. P. (2009). Gerencia Logica y Global. Universidad EAN. 
Villareal, S. F. (2012). Logistica Integral ; Una alternativa para crear valor y ventaja competitiva en las pequeñas y medianas empresas (pymes) Sector calzado. Nova Scientia, 169. 